\title{
Reprogrammation fonctionnelle de gamètes mâles par des embryons haploïdes parthénotes
}

Laure Coulombel

> Dans son édition du 13 septembre 2016, le journal Le Monde publie un article intitulé «Des chercheurs sont parvenus à reproduire des souris sans recourir à des ovocytes » [1] rendant compte des travaux d'équipes du Royaume-Uni et d'Allemagne publiés dans Nature Communications [2]. Ce titre accrocheur joue sur les mots, mais il est tout à fait inexact; il est d'ailleurs surprenant que ces travaux aient retenu l'attention de la grande presse, car ils ont peu d'implications immédiates dans le domaine de la procréation humaine ; il s'agit avant tout d'un travail de recherche fondamentale. De quoi s'agit-il? D'explorer si un ovocyte (gamète femelle) qui a terminé la seconde méiose et s'est engagé dans le processus de transition vers un processus de divisions mitotiques embryonnaires, peut encore reprogrammer le génome du spermatozoïde pour former un zygote totipotent capable d'assurer le développement d'un individu viable.

\section{La fécondation : quelques rappels}

la fécondation est le processus par lequel le noyau du gamète mâle haploïde (n) - le spermatozoïde - au génome extrêmement compacté, et l'ovocyte haploïde (n) - gamète femelle - dans lequel il pénètre, forment un zygote totipotent diploïde $(2 n)$, capable de se développer en un individu viable (Figure lA). Lors de la fécondation, l'ovocyte est bloqué en métaphase II de la seconde méiose (il est haploïde), et c'est la pénétration du spermatozoïde (également haploïde) qui déclenche, en partie via un signal calcique, la levée du blocage et l'achèvement de la méiose. Le zygote 1-cellule ainsi formé s'engage alors dans un processus mitotique classique mais sans activité transcriptionnelle. La première division mitotique donne naissance à deux «blastomères » (un embryon 2-cellules) (Figure $1 A$ ). À ce stade, la transcription du génome embryonnaire débute. Ce processus requiert une reprogrammation très complexe de la structure des noyaux des gamètes - cellules post-mitotiques très différenciées - qui met en jeu des modifications épigénétiques majeures impliquant notamment des échanges d'histones, des modifications chimiques des histones, et une déméthylation globale des génomes maternel et paternel. L'ovocyte au stade de métaphase II de la méiose a donc ce potentiel étonnant de reprogrammer un noyau de spermatozoïde ; on connaît aussi son potentiel de reprogrammer le noyau d'une cellule somatique en un zygote totipotent après un «transfert nucléaire » (ou « clonage thérapeutique »).

La question que posent les auteurs de cet article est la suivante : un ovocyte à un stade autre que celui de métaphase Il de la méiose serait-il capable d'induire la reprogrammation d'un gamète mâle pour former un embryon? Plus précisément, un ovocyte dans une des phases du cycle cellulaire mitotique
Directrice de recherche émérite, Inserm U1130, conseillère scientifique de médecine/sciences, Paris, France. laure.coulombel@inserm.fr

(donc au stade de zygote ou d'embryon) et non du cycle de la méiose, en serait-il capable? Au-delà, pourrait-on imaginer qu'une cellule autre qu'un ovocyte puisse avoir ce pouvoir? On sait que des noyaux issus d'embryons 2-ou 8-cellules, ou des noyaux de cellules souches embryonnaires ${ }^{1}$, peuvent être reprogrammés efficacement s'ils sont transférés dans des ovocytes en prométaphase I, ou dans des zygotes mitotiques énucléés, mais que cette activité décroît rapidement après fécondation ou activation ovocytaire [3-5]. Qu'en est-il dans ces conditions de la reprogrammation de spermatozoïdes, dont le noyau est très différent de celui de noyaux somatiques, en particulier par sa chromatine extrêmement compacte, la présence de protamines et l'absence de synthèse d'ADN ? Un groupe de Shanghai avait déjà décrit une reprogrammation partielle du sperme mature, plus efficace avec des spermatides, par des blastomères haploïdes issus de parthénotes, sans toutefois aboutir à la naissance d'animaux viables [6].

\section{Reprogrammation de sperme par des zygotes parthénotes haploïdes}

Les auteurs ont testé la capacité de parthénotes de reprogrammer des

\footnotetext{
${ }^{1}$ Ces noyaux embryonnaires sont pluripotents, et leur organisation chromatinienne nucléaire - assez proche de celle de noyaux d'embryons précoces - reste encore facilement modulable.
} 
gamètes mâles. Rappelons que des ovocytes haploïdes en métaphase II de la méiose peuvent, lorsqu'ils sont stimulés artificiellement (chlorure de strontium), sortir de la méiose et entrer dans un processus de divisions mitotiques embryonnaires (embryons parthénogénétiques) (Figure $I B$ ). $\varepsilon n$ l'absence de fécondation par du sperme, un embryon parthénogénétique, qui ne contient qu'un seul jeu de chromatides, issus de la mère, peut se développer jusqu'au stade blastocyste, mais ne donnera pas d'individu viable après transfert dans l'utérus, en l'absence de contribution génomique paternelle. Des lignées de cellules ES (embryonic stem cells) haploïdes, dérivées de ces blastocystes, et que l'on sait depuis peu maintenir en culture sous forme haploïde, sont fort utiles pour tester les conséquences de mutations récessives [7]. C'est dans ces embryons parthénogéniques, très tôt après l'activation ovocytaire, à des temps variables (7, 10 ou 13 heures, désignés phICSI-13), que les auteurs ont injecté du sperme - selon la technique dite d'ICSI (intracytoplasmic sperm injection) (Figure IB). Globalement, $35 \%$ des embryons se développent jusqu'au stade blastocyste, proportion qui atteint $55 \%$ si l'ICSI est réalisée 7 ou 10 heures après l'activation ovocytaire. Des gènes-étiquettes permettent de repérer l'activation du génome paternel, et d'identifier le caractère - uniparental ou biparental - des blastomères des embryons. Globalement, de 1 à $8 \%$ des embryons transférés (1 108 au total) dans l'utérus se sont développés en des souriceaux vivants et fertiles. Seules des cellules biparentales étaient détectées à la naissance, alors que le génome de certains blastomères des embryons plus précoces était uniparental. Les auteurs ont également testé la capacité de ces embryons 2-cellules parthénogénétiques à reprogrammer non pas du sperme mature, mais des spermatides rondes (un stade plus immature du développement de ces gamètes ${ }^{2}$, qui notamment ne contient pas de protamines). Le taux de naissances d'animaux viables et fertiles était de 2,6\% (32\% pour les contrôles, chez lesquels I'ICSI est réalisée dans les ovocytes en méiose). Comme précédemment, le taux de succès dépend du temps écoulé entre la rupture de la membrane nucléaire de l'ovocyte stimulé et l'injection des gamètes mâles. Ces deux types d'expériences démontrent que la machinerie mitotique de zygotes l-cellule est capable de remodeler la structure chromatinienne de certains spermatozoïdes matures comme de spermatides immatures.

\section{Qualité de la reprogrammation des gamètes mâles par des zygotes mitotiques}

Outre l'analyse fonctionnelle - développement embryonnaire et naissance à terme d'animaux viables - les auteurs ont aussi recherché les stigmates moléculaires d'une reprogrammation du génome paternel dans les embryons obtenus après une procédure d'ICSI dans des zygotes parthénotes (voir plus loin). $\varepsilon$ n effet, lors d'une fécondation « naturelle » ou après ICSI dans un ovocyte en méiose, la chromatine des noyaux de l'ovocyte et des gamètes mâles se remodèle simultanément $[8,9]$. Mais lorsque le sperme ou les spermatides sont injectés plus tard en phase mitotique des ovocytes activés, il y a un asynchronisme temporel important dans la mise en place des modifications des structures chromatiniennes gamétiques mâle et femelle. Or, la complexité et la cinétique de ce processus sont encore très mal connues, notamment les étapes qui interviennent lors de la transition méiose-mitose (environ $13 \mathrm{~h}$ Figure $1 \mathrm{~A}$ ), potentiellement importantes pour la reprogrammation harmonieuse

\footnotetext{
${ }^{2}$ Notons que certaines procédures d'ICSI chez l'homme consistent à injecter aussi des spermatides immatures dans l'ovocyte (notamment dans les dysfonctionnements de la différenciation des gamètes).
}

du noyau de gamète mâle. De telles phases sont court-circuitées lorsque les gamètes mâles sont injectés au stade d'embryons parthénotes 1 ou 2-cellules. L'importance de cette temporalité a été testée indirectement dans l'article: les auteurs ont transféré dans des blastomères haploïdes (embryons parthénotes stade 2-cellules), $30 \mathrm{~min}$ après la première division mitotique, le pronucléus mâle de zygotes obtenus par une ICSI classique (pronucléus prélevé $4 \mathrm{~h}$ après ICSI). Aucun animal viable n'a été obtenu.

Sans entrer dans les détails de l'analyse moléculaire des critères de reprogrammation des noyaux des gamètes, les profils transcriptionnels et épigénétiques des embryons obtenus après ICSI dans des blastomères haploïdes mitotiques sont différents de ceux d'embryons obtenus par une procédure classique d'ICSI (ICSI dans l'ovocyte métaphase II de la méiose), sans que l'implication précise de ces différences soit encore clairement établie. Parmi ces stigmates de reprogrammation, mentionnons l'élimination des protamines (protéines basiques qui remplacent les histones dans les gamètes mâles en fin de maturation, et sont éliminées précocement après la fécondation et remplacées par des histones ovocytaires pour former le pronucléus mâle), la caractérisation d'histones canoniques ou de variants les plus étudiés de la chromatine paternelle, et les modifications épigénétiques à type d'acétylation (H4Kl2ac) et de méthylation (H3K4, H3K27, H3K9) de ces histones. Second niveau de régulation, I'ADN paternel lui-même, qui subit une déméthylation globale et active très rapide dès le stade 1 -cellule (perte de $5 \mathrm{mC}$ [5-méthylcytosine], oxydation de $5 \mathrm{mC}$ en 5 -hydroxyméthylcytosine [5hmC] par l'enzyme Tet3). Or, dans les embryons parthénotes ayant subi une ICSI par référence à des embryons ICSI contrôles, la dynamique de régulation des taux paternels versus maternels de $5 \mathrm{hmC}$ et de $5 \mathrm{mC}$ est perturbée. Les causes et les conséquences de ces ano- 


\section{A Fécondation en phase de méiose}

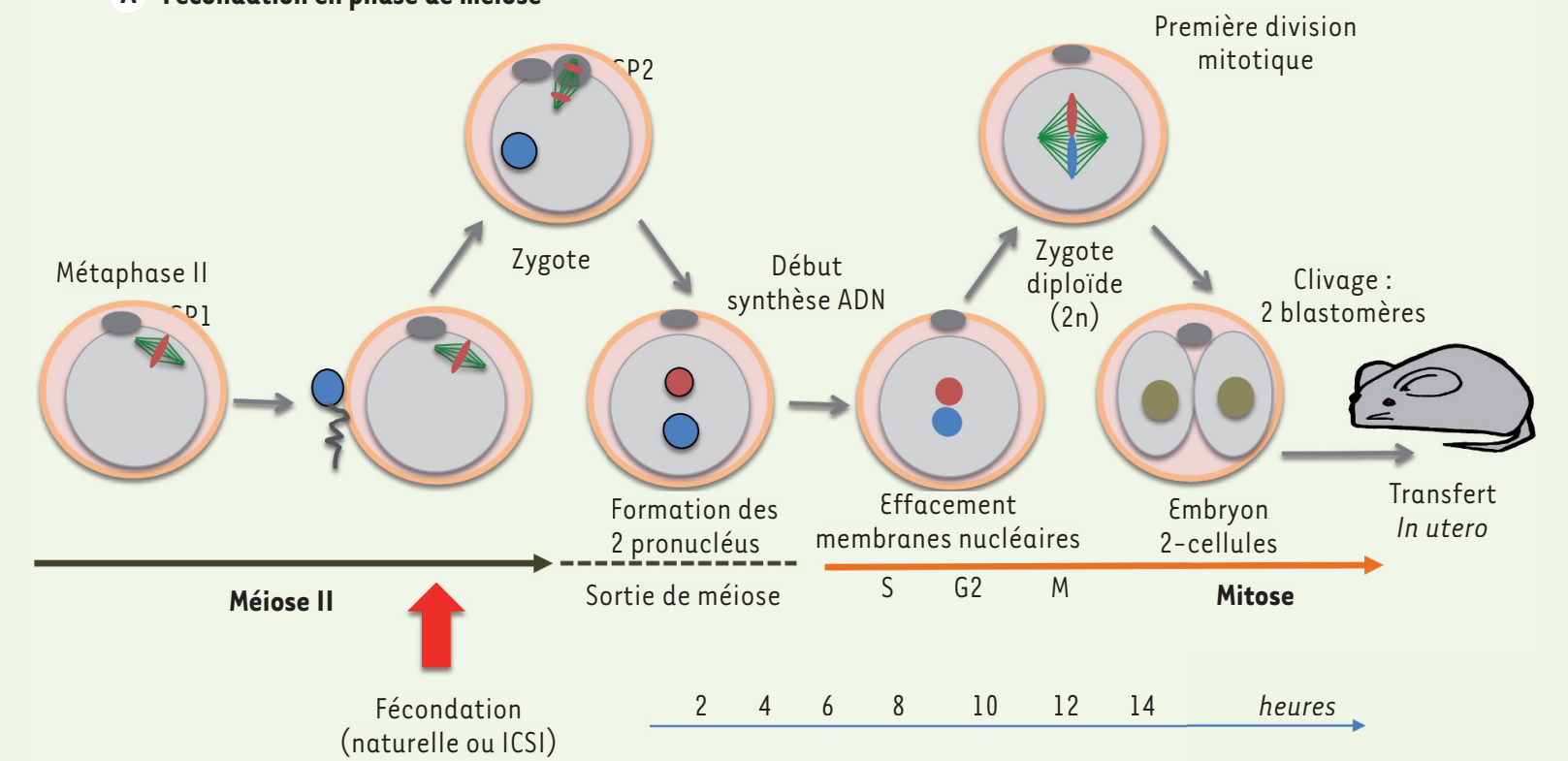

B Injection du sperme dans un zygote en mitose

Première division
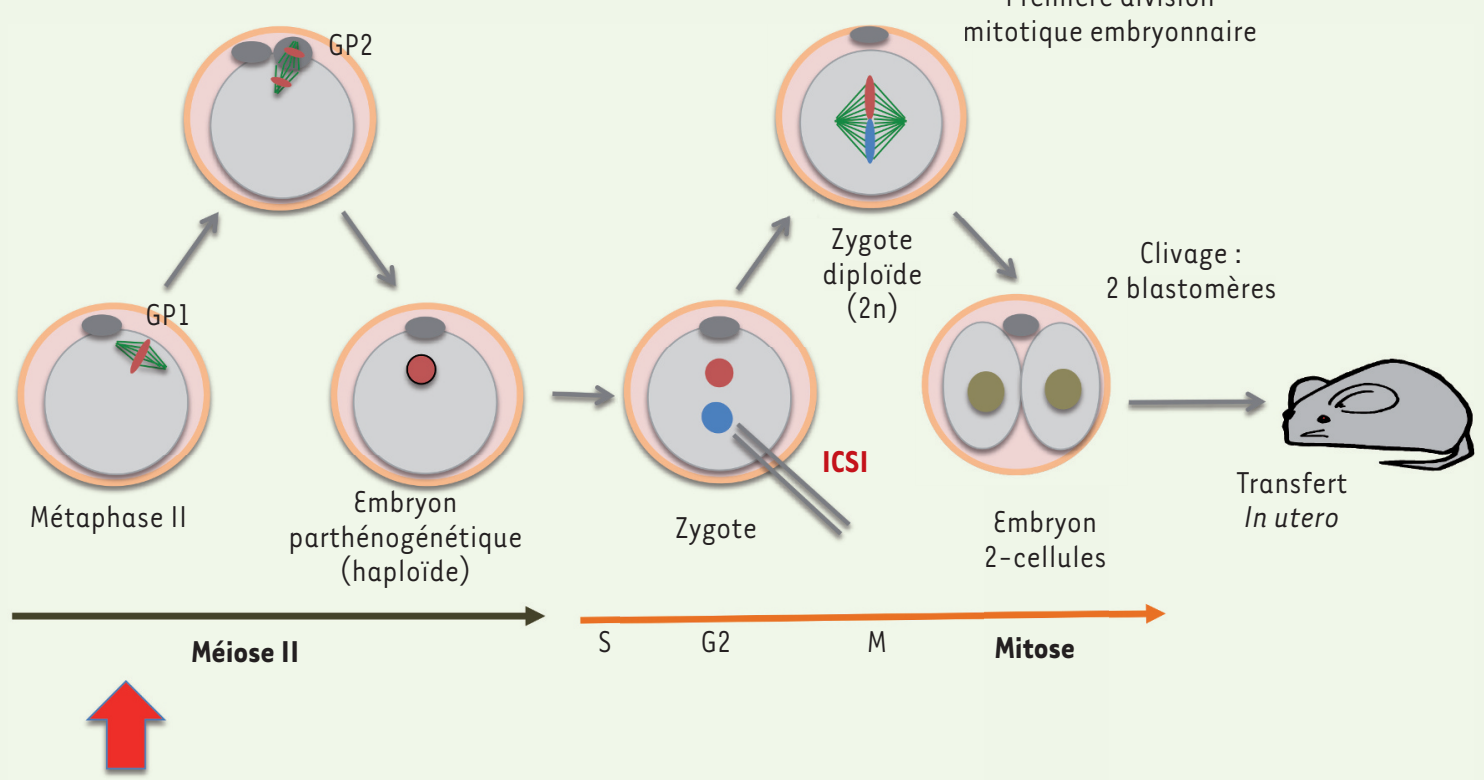

Activation par $\mathrm{SrCl} 2$

(chlorure de strontium)

Figure 1. Procédure expérimentale d'ICSI pendant la phase de méiose (A) et de mitose (B). A. La fécondation (naturelle ou via une procédure d'ICSI) survient dans un ovocyte arrêté en métaphase de la méiose 2. L'entrée du sperme déclenche l'achèvement de la méiose, avec l'expulsion du second globule polaire (GP2), puis la formation des deux pronucléus (haploïdes, ln) mâle et femelle distincts et le début de la synthèse d'ADN dans ces deux noyaux. C'est la transition vers le processus de mitose et la formation d'un zygote diploïde (2n), qui effectue une première division mitotique conduisant à un embryon composé de deux blastomères. La cinétique de ce processus est indiquée (chez la souris). B. La procédure expérimentale utilisée dans cet article consiste à différer l'injection du sperme jusqu'à la phase de début de mitose. L'ICSI est réalisée dans un zygote haploïde, obtenu par l'activation artificielle ( $\mathrm{SrCl} 2)$ d'un ovocyte en métaphase de méiose 2. Cette activation enclenche l'expulsion du second globule polaire, la sortie de méiose et la transition méiose-mitose. Le délai entre l'activation de l'ovocyte et l'ICSI (7, 10 ou 13 heures) est déterminant. 
malies significatives de l'hydroxyméthylation de la méthylcytosine du génome paternel ne sont toutefois pas élucidées, et doivent être interprétées à la lumière des très récents développements du mécanisme de la déméthylation de I'ADN du zygote $[10,11]$. Des différences transcriptomiques ont également été identifiées entre les embryons ICSI et phICSI-13, en particulier pour 73 gènes, dont, de façon notable, des gènes maîtres de la pluripotence, dont le défaut d'expression explique probablement l'arrêt de développement au stade de morula de certains embryons.

\section{Quel message scientifique?}

Le résultat principal de cette étude est que des ovocytes activés artificiellement vers un processus de parthénogenèse gardent la capacité de reprogrammer l'organisation chromatinienne de gamètes mâles, immatures (spermatides) ou matures (spermatozoïdes), et de former des blastomères diploïdes se développant dans une proportion non négligeable de cas en des animaux viables et fertiles. Et ceci malgré l'identification de différences épigénétiques et transcriptomiques dans tous ces embryons comparés avec des embryons ICSI contrôles. Le lien temporel entre la phase du premier cycle cellulaire mitotique une fois la méiose complétée pendant laquelle l'ICSI est réalisée, et le succès du développement embryonnaire à terme suggère qu'existe une fenêtre permissive très étroite lors de la transition méiose-mitose, correspondant peut-être à la métaphase de la première mitose. Ceci est aussi corroboré par la cinétique des expériences de transfert dans des embryons parthénotes du pronucléus mâle prélevé après une ICSI classique.

De fait, certains des composants ovocytaires et des cofacteurs individuellement requis pour le remodelage du noyau de spermatozoïdes (et en particulier la décondensation de l'ADN) semblent présents dans les cellules mitotiques.
Rappelons que des ovocytes comme des embryons 2-cellules haploïdes ont été montrés comme pouvant reprogrammer des noyaux de spermatides (gamètes mâles immatures) ainsi que des noyaux somatiques.

\section{Une conclusion éthique un peu bâclée}

Les données de cet article font progresser avant tout la compréhension du mécanisme de reprogrammation à l'œuvre lors de la fécondation gamétique chez le rongeur, mais elles n'ont pas d'implications évidentes en clinique humaine. Les auteurs terminent pourtant par une digression éthique qui devrait, à notre avis, être mieux documentée pour être convaincante: peut-on en effet suggérer que la seule observation qu'un zygote en phase mitotique induit la reprogrammation de gamètes mâles suffit à obscurcir la frontière fonctionnelle entre embryon, gamètes, et cellules somatiques? II est exact que les ovocytes, les zygotes mitotiques et les cellules ES expriment une activité de reprogrammation de la structure chromatinienne qui s'exerce à des degrés divers sur différentes cellules, suggérant la persistance de cette fonctionnalité de l'ovocyte à la cellule pluripotente. Mais, peut-on, au vu de la procédure très artificielle utilisée dans cette étude, remettre en question les réflexions juridique et éthique sur la dérivation de cellules ES à partir de blastocystes issus de parthénotes, dont celle de la Cour Européenne de Justice ${ }^{3}$, qui concluait à la non-équivalence des cellules $E S$ issues de parthénotes et de celles issues de

\footnotetext{
3 L'article 6, paragraphe 2, sous c), de la directive 98/44/ C $\varepsilon$ du Parlement européen et du Conseil, du 6 juillet 1998 , relative à la protection juridique des inventions biotechnologiques, doit être interprété en ce sens qu'un ovule humain non fécondé qui, par voie de parthénogenèse, a été induit à se diviser et à se développer ne constitue pas un « embryon humain », au sens de cette disposition, si, à la lumière des connaissances actuelles de la science, il ne dispose pas, en tant que tel, de la capacité intrinsèque de se développer en un être humain, ce qu'il appartient à la juridiction nationale de vérifier.
}

la masse interne d'un embryon issu d'une fécondation gamétique et conçu initialement dans le cadre d'un projet parental ? Bien au contraire puisque les auteurs démontrent des profils transcriptionnels et épigénétiques distincts, même si dans un très petit nombre de cas (moins de $10 \%$ ) le résultat final (c'est-à-dire la naissance d'un souriceau viable), semble identique. Le faible taux de succès pourrait par exemple s'expliquer par une ségrégation asymétrique des facteurs essentiels à la reprogrammation, certains ovocytes parthénogénotes retenant ainsi les mêmes propriétés que l'ovocyte source. Les premières étapes du développement embryonnaire sont parmi les processus biologiques les plus complexes, et les avancées spectaculaires dans leur connaissance et leur manipulation méritent mieux que des conclusions à l'emporte-pièce qui n'élaborent pas la complexité. La prudence et le recul devraient d'autant plus être de mise qu'affleure la question vertigineuse de l'obtention de gamètes «synthétiques » ou d'une possible duplication dans des cellules autres que des ovocytes du pouvoir de reprogrammation en cellules totipotentes. $\diamond$

Mitotic reprogramming of sperm injected into haploid

parthenogenotes embryos

\section{REMERCIEMENTS}

Merci à Marie-Hélène Verlhac pour sa relecture critique du texte et son aide pour la figure.

\section{LIENS D'INTÉRÊT}

L'auteur déclare n'avoir aucun lien d'intérêt concernant les données publiées dans cet article.

\section{RéFÉRENCES}

1. En savoir plus sur http://www.lemonde.fr/ medecine/article/2016/09/13/des-chercheurssont-parvenus-a-reproduire-des-souris-sansrecourir-a-des-ovocytes_4997176_1650718. html\#EfD0OVfOCBmp34SI.99

2. Suzuki T, Asami M, Hoffmann M, et al. Mice produced by mitotic reprogramming of sperm injected into haploid parthenogenotes. Nat Comm 2016 ; 7 : 12676. 


\section{RÉFÉRENCES}

3. Yang H, Shi L, Degui Chen C, Li J. Mice generated after round spermatid injection into haploid twocell blastomeres. Cell Res $2011 ; 21: 854-8$.

4. Kang $\varepsilon, W u G, M a H$, et al. Nuclear reprogramming by interphase cytoplasm of two-cell mouse embryos. Nature $2014 ; 509$ : 101-4

5. Egli D, Rosains J, Birkhoff G, Eggan K. Developmental reprogramming after chromosome transfer into mitotic mouse zygotes. Nature 2007 $447: 679-85$
6. Wakayama T, Tateno H, Mombaerts $P$, Yanagimachi R. Nuclear transfer into mouse zygotes. Nat Genet $2000 ; 24: 108-9$.

7. Wutz A. Haploid mouse embryonic stem cells: rapid genetic screening and germline transmission. Annu Rev Cell Devel Biol 2014 ; 30 : 705-22

8. Yartseva V, Giraldez AJ. The maternal-to-zygotic transition during vertebrate development: a model for reprogramming. Curr Top Dev Biol $2015 ; 113$ : 191-232.

9. Lee MT1, Bonneau AR, Giraldez AJ. Zygotic genome activation during the maternal-to-zygotic transition. Annu Rev Cell Dev Biol 2014 ; 30 : 581-613.
10. Messerschmidt DN. A twist in zygotic reprogramming. Nat Cell Biol 2016; $18: 139-40$.

11. Amouroux R, Nashun B, Shirane K, et al. De novo DNA methylation drives $5 \mathrm{hmC}$ accumulation in mouse zygotes. Nat Cell Biol $2016 ; 18: 225-33$.

\section{TIRÉS À PART}

L. Coulombel

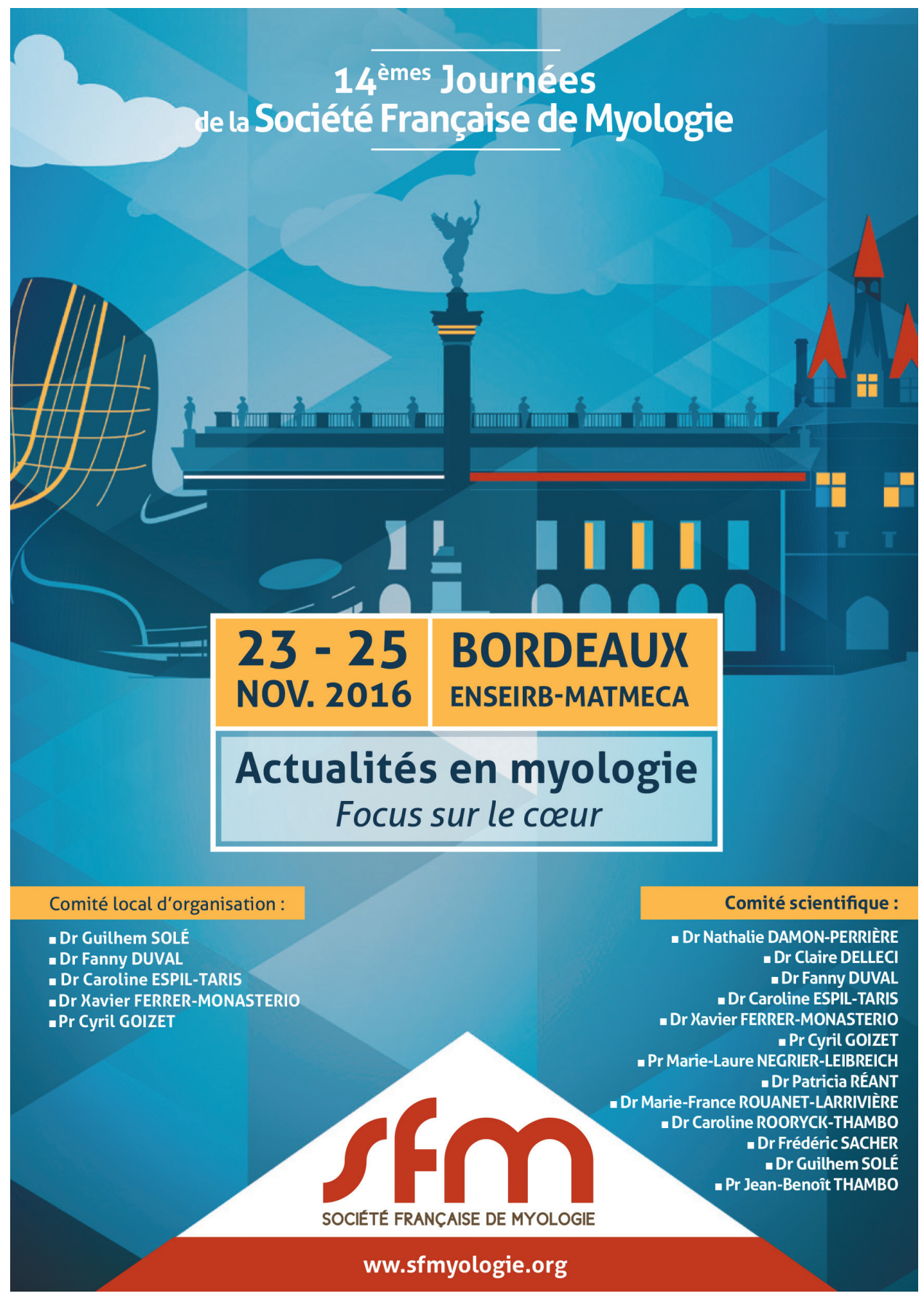

